\title{
MATHEMATIZING THE UNIVERSE
}

By Rodney Bartlett

Abstract -

I think the best way to describe this article is by listing the

CONTENTS -

INTRODUCTION - Speculations about life, the universe and everything which have a basis in science

SECTION 1 - STEFAN-BOLTZMANN LAW, RADIATION PRESSURE FROM THERMODYNAMICS, "VECTOR-TENSOR-SCALAR GEOMETRY" ANALOGOUS TO STRESS TENSOR / EIGENVECTORS / EIGENVALUES

SECTION 2 - VECTOR-TENSOR-SCALAR GEOMETRY AND THE NUCLEAR FORCES

SECTION 3 - THE STRESS TENSOR ON THE COSMIC LEVEL

SECTION 4 - BITS AND TOPOLOGY - CONTINUING THE THEME OF THE MOBIUS STRIP AND MATHEMATICAL MATTER

SECTION 5 - ANALOGY OF QUANTUM SPIN AND MATRIX ARRAY, WITH ELECTROMAGNETIC AND GRAVITATIONAL WAVES PRODUCED FROM PURE MATHEMATICS

SECTION 6 - DARK MATTER, DARK ENERGY AND A HIGHER DIMENSION OF SPACE-TIME

SECTION 7A - EXPLAINING OCEAN TIDES WHEN GENERAL RELATIVITY SAYS GRAVITY IS A PUSH CAUSED BY THE CURVATURE OF SPACE-TIME

7B - M-SIGMA

7C - GEYSERS ON SATURN'S MOON ENCELADUS

7D - A BRIEF HISTORY OF GRAVITY 


\section{INTRODUCTION - Speculations about life, the universe and everything which have a basis in science}

In a science TV program [Australian Broadcasting Corporation, 'Custom Universe Finetuned For Us?", https://www.abc.net.au/catalyst/stories/3836881.htm (2013), Dr. Graham Phillips reported,

'the physicist and writer Paul Davies thinks the universe is indeed finetuned for minds like ours. And who finetuned it? Not God but minds from the future, perhaps even our distant descendants, that have reached back through time ... and selected the very laws of physics that allow for the existence of minds in the first place. Sounds bizarre, but quantum physics actually allows that kind of thing.'

And Carl Sagan writes [Carl Sagan, 'Pale Blue Dot - A Vision of the Human Future in Space' (Headline Book Publishing, 1995)],

'Many religions, from Hinduism to Gnostic Christianity to Mormon doctrine, teach that as impious as it may sound - it is the goal of humans to become gods.'

Author comment: maybe the term God should be redefined to mean 'our distant descendants, that have reached back through time ...' Their population of billions would know how to apply the laws of Quantum Gravity - the anticipated unification of Quantum Mechanics and Einstein's theory of gravity, General Relativity - to become omnipresent in space-time as well as other dimensions, and to become omnipotent and omniscient. They could then constitute the Elohim - a name used for God in the Old Testament which, according to World Book Encyclopedia, means the PLURAL MAJESTY OF THE ONE GOD.) ['Elohim', The World Book Encyclopedia (Field Enterprises Educational Corporation, 1967) 
It seems possible that, after death and before conception, a human exists as a member of the Elohim: humans from the distant future who have learned how to affect all space and all time, including our past and present. Since anything and everything is possible for such a being, we could either exist eternally in that condition or choose to be born on Earth and have a human life. The latter would give us new perspective and experiences. It would also allow us to directly contribute to the eventual rise of Elohim civilization: perhaps by adding something to some field of knowledge or technology (this might oneday lead to the ability to choose eternal life as a human); perhaps by ensuring that the human race continues into new generations; perhaps by sharing with, and otherwise helping, whomever we can.

The Elohim use Quantum Gravity and the Theory of Everything to become entangled with all space-time. Therefore, their power has no limits and it must be possible for them to concentrate those abilities in, and to occupy, one individual body (a part of the space-time they're entangled with). That individual (and since the entire universe, the present, all the past, and all the future are united; every individual) has the potential to be omnipresent, omnipotent, and omniscient. One day, you and I will fulfil our potential to exist everywhere and everywhen (this could be termed immortality). We'll also someday be all-powerful and all-knowing. Regarding a more limited and present-day application of the latter, I think something written by Stephen Hawking points the way to amateurs making useful contributions to theoretical physics and mathematics. Let me explain:

Hawking wrote,

"If a complete unified theory was discovered, it would only be a matter of time before it was digested and simplified and taught in schools, at least in outline. We should then all be able to have some understanding of the laws that govern the universe and are responsible for our existence." [Stephen Hawking, A Brief History of Time (Bantam Press, 1988)]

My comment: A complete unified theory would not be restricted to mathematics, for that would make the theory incomplete. A complete theory would, by definition, affect 
everything in space-time. Affecting everything in time means future generations (and even what you and I would call past or present generations) would be able to learn how to intuitively access the knowledge of future centuries. Unification necessarily means today's scientific approach of viewing objects and events as separate will become limited to the way senses perceive objects and events. Separateness will belong to 'classical' existence, and unification to 'quantum mechanical' existence where all energy and matter, objects and events in space-time are entangled.

I think we rely too much on the idea of biological evolution. Obviously, evolution does exist - we see it all the time when we study how living things adapt. But that doesn't mean it can be extrapolated to explain everything about life (sorry, Mr. Darwin, but I can't see it explaining the origins of life).

A model of the cosmos might be built that uses pi and imaginary time, and resides in Virtual Reality (artificial, computer-generated simulation). The entanglement (quantummechanics style) in the simulated universe is unable to remain separate from the entanglement existing in our perceived reality because computers using so-called "imaginary" time (which is defined by numbers with the property $i^{2}=-1$ ) removes all boundaries between the two universes. This enables them to become one Augmented Reality (known now as technology that layers computer-generated enhancements onto an existing reality, but seen here as the related layering of virtual reality onto other points in time and space). The poorly-named imaginary time of physics and mathematics unites with pi (both are necessary to generate an infinite universe: alone, unbounded imaginary time is finite).

As suggested by Elon Musk (founder of a number of high profile companies, such as Tesla and Space X):

'If you assume any rate of improvement at all, then the games will become indistinguishable from reality, even if that rate of advancement drops by a thousand from what it is now. Then you just say, okay, let's imagine it's 10,000 years in the future, which is nothing on the evolutionary^ scale. So given that we're clearly on a trajectory to have games that are indistinguishable from reality, and those games could be played on any set-top box or on a PC or whatever, and there would probably be billions of such computers or set-top boxes, it would seem to follow that the odds that we're in base (non-simulated) reality is one in billions.' (The Conversation, 'Elon Musk says we're probably living in a computer simulation - here's the science', https://theconversation.com/elon-musk-says-were-probably-living-in-a-computersimulation-heres-the-science-60821 (2016)) 
${ }^{\wedge}$ Evolution doesn't $100 \%$ compute, according to this post. Evolution would always exist in the forms of adaptation and of modification to anatomy/physiology, but it would not explain origins. Consider the future revolution of time travel combined with the unimaginable biotechnology and genetic engineering of centuries to come. Isn't it conceivable that plants, animals and even humans are the product of entirely natural intelligent design by humanity of the distant future? Making production a two-way process is the fact that humans of the distant future rely on the reproductive instincts of past and present men and women for their existence.

Prof. Stephen Hawking says that boundaries and singularities exist in real time but don't exist in imaginary time. (Stephen Hawking, A Brief History of Time (Bantam Press, 1988), p. 139) There really are boundaries in real time and it must hypothetically be possible to step outside the universe if only real time exists. But when so-called imaginary time also exists, it is not possible to step outside the universe because the boundaries simply aren't there and the universe has no end or start (neither in space nor in time). Only one universe can then exist, and there is no multiverse.

I guess all this (not only the origin of life, but also the origin of the universe) sounds like science fiction. But if you tried to explain today's world to someone from 200 years ago, it would all sound like pure science fiction to just about everybody. 200 years from now, there might be nothing fictional about what l've written. It might even sound unimaginative to the great-grandkids of your great-grandkids.

\section{STEFAN-BOLTZMANN LAW, RADIATION PRESSURE FROM THERMODYNAMICS, "VECTOR-TENSOR-SCALAR GEOMETRY" ANALOGOUS TO STRESS TENSOR / EIGENVECTORS / EIGENVALUES}

(background info in 1st 3 paragraphs from Wikipedia - last 2 paragraphs extrapolate from historical background to possible modern application)

A blackbody is an idealized physical body that absorbs all incident electromagnetic radiation, regardless of frequency or angle of incidence. The Stefan-Boltzmann law states that the total energy radiated is directly proportional to the fourth power of the black body's temperature in degrees Kelvin. The kelvin is not normally referred to or written as a degree but is often used in conjunction with the degree Celsius, which has 
the same magnitude. It's named after engineer and physicist William Thomson, 1st Baron Kelvin (1824-1907) and uses absolute zero (-273.15 degrees Celsius) as its starting point.

In 1864, John Tyndall presented measurements of the infrared emission by a platinum filament and the corresponding color of the filament. The proportionality to the fourth power of the absolute temperature was deduced by Josef Stefan (1835-1893) in 1879 on the basis of Tyndall's experimental measurements. A derivation of the law from theoretical considerations was presented by Ludwig Boltzmann (1844-1906) in 1884, drawing upon the work of Adolfo Bartoli. Bartoli in 1876 had derived the existence of radiation pressure from the principles of thermodynamics - the branch of physics that deals with heat and temperature; plus their relation to energy, work, radiation, and properties of matter.

In 1874 James Clerk Maxwell found out that the existence of tensions in the ether, in other words radiation pressure, follows from his electromagnetic theory. In 1876 Bartoli derived the existence of radiation pressure from thermodynamics. He argued that the radiant temperature of a body can be raised by, say, reflecting its light back on to itself from a mirror, adding heat which warms it - and therefore it is possible to transport energy from the colder mirror to the hotter body (itself). To avoid this violation of the second law of thermodynamics, it is necessary that light impart a pressure to the mirror. Therefore, the radiation pressure was also called "Maxwell-Bartoli pressure". Later the radiation pressure played an important role in the work of Albert Einstein in connection with mass-energy equivalence and the photoelectric effect.

As will be seen later in this paragraph, the concept of radiation pressure can be applied to what I call "vector-tensor-scalar geometry" because "the energy and momentum of the photons and presently hypothetical gravitons exert a pressure we call mass". Two adjoining sides of a parallelogram represent the vectors of the photon's spin 1 and the graviton's spin 2. The resultant diagonal represents the interaction of the sides/vectors $(1 \div 2=$ the spin $1 / 2$ of every matter particle). Tensor calculus changes the coordinates of the sides into the coordinates of a position on a line (a single point on the diagonal). This scalar point is associated with particles of spin 0 . If the mass produced during the $1 \div 2$ interaction - the energy and momentum of the photons and presently hypothetical gravitons exert a pressure we call mass - happens to be $125 \mathrm{GeV} / \mathrm{c} 2$, its union with spin 0 produces the Higgs boson. $125 \mathrm{GeV} / \mathrm{c} 2$ united with spin 0 means the central scalar point of the Higgs boson is related to the vector of the graviton's spin 2, and the Higgs field is therefore united with the supposedly unrelated gravitational field (together with the latter's constant interaction with the electromagnetic field).

Stress may be defined, in this case, as a physical quantity that expresses the 
continuous internal pressure that photon and graviton particles exert during the thermodynamical process of formation of properties of matter. Combining this with tensor calculus changing the coordinates of the sides into the coordinates of a position on a line (the diagonal) could change the term "vector-tensor-scalar geometry" into the term "stress tensor". The geometry's two adjoining sides of a parallelogram may be thought of as eigenvectors ("eigen" is a German word meaning "characteristic") of a transforming, nonzero nature that produce the eigenvalues of the diagonal, which include the scalar Higgs boson as well as other values such as the spin 1 bosons of the strong/weak nuclear forces.

\section{VECTOR-TENSOR-SCALAR GEOMETRY AND THE NUCLEAR FORCES}

One of parallelogram's horizontal sides represents the electromagnetic component

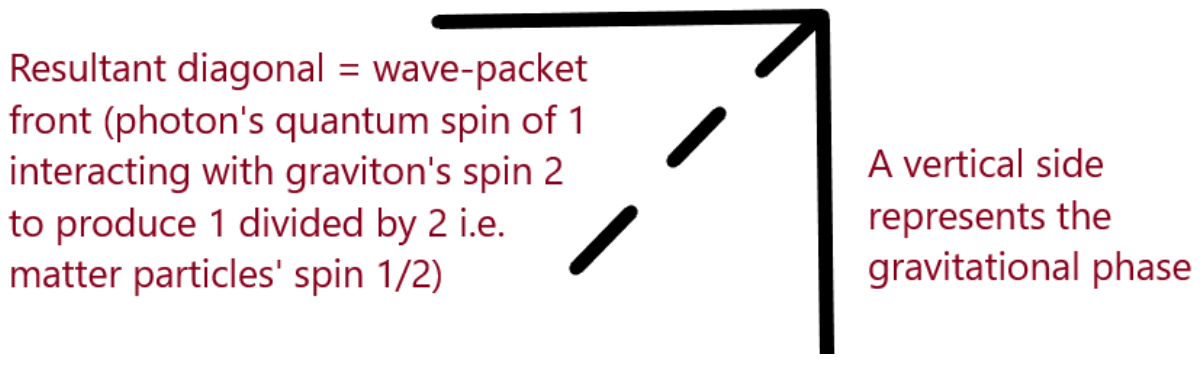

FIGURE 1: WAVE-PACKET FRONT AS QUANTUM SPIN

It must be remembered that referring to space alone is incomplete. Living in space-time, it's necessary to add some sentences about the time factor. The photon must interact with the graviton to produce the mass of the mass of the weak nuclear force's W and $Z$ bosons. To produce their quantum spin, the photon's spin 1 needs to react with the graviton's spin 2. That is, the photon's turning through one complete revolution needs to be combined with the graviton's being turned through two half-revolutions. ${ }^{\wedge}$

Incorporating the time factor as a reversal of time in the middle of the interaction: a gravitonic half revolution is subtracted from the photonic full revolution then the graviton's time-reversal adds a half revolution $(1-1 / 2+1 / 2=$ the spin 1 of $W$ and $Z$ bosons). The strong nuclear force's gluon's quantum spin of 1 could arise in the same way as the spin 1 of weak-force bosons. 
^ Professor Stephen Hawking writes,

"What the spin of a particle really tells us is what the particle looks like from different directions." (Stephen Hawking, 1988, 'A Brief History of Time', pp.66-67 - Bantam Press)

Spin 1 is like an arrow-tip pointing, say, up. A photon has to be turned round a full revolution of 360 degrees to look the same.

Spin 2 is like an arrow with 2 tips - 1 pointing up, 1 down. A graviton has to be turned half a revolution (180 degrees) to look the same.

Spin 0 is like a ball of arrows having no spaces. A Higgs boson looks like a dot: the same from every direction.

Spin $1 / 2$ is logically like a Mobius strip, though Hawking doesn't specifically say so. This is because a particle of matter has to be turned through two complete revolutions to look the same, and you must travel around a Mobius strip twice to reach the starting point.

\section{THE STRESS TENSOR ON THE COSMIC LEVEL}

The interacting gravity and electromagnetism produce mass e.g. they can form a Higgs boson or the weak nuclear force's bosons as well as matter. On a cosmic level - if gravitational and electromagnetic waves focus on a protoplanetary disk surrounding a newborn star, the quantum spin of the particles of matter in the disk (1 / 2) could imprint $^{\wedge}$ itself on the waves' interaction and build up a planet layer by layer from vectortensor-scalar geometry's $1 \div 2$ interaction. This method of building planets is preferred to collisions between rocks and dust in the disk because most planetary systems seem to outweigh the protoplanetary disks in which they formed, leaving astronomers to reevaluate planet-formation theories. If the waves focus on a region of space where there's no matter, the opposite interaction occurs and the graviton's spin 2 is divided by the photon's spin 1 to produce $2 \div 1$. The mass produced has the spin inherent in each of the gravitons composing the body of gravitational waves, and could be an alternative method to supernovas for producing black holes. 
^ What do I mean by "imprint"? To explain using something different - when a laser scanner reads a barcode, the information in the barcode is "imprinted" in the scanner. When gravitational and electromagnetic waves scan a protoplanetary disk, the info in the disk is recorded or imprinted in the waves. When they scan "empty" space-time, the info pertaining to the graviton's spin of $2(2 / 1)$ is imprinted since gravity is space-time according to General Relativity: it says gravity is just another term for the curvature of space-time.

\section{BITS AND TOPOLOGY - CONTINUING THE THEME OF THE MOBIUS STRIP AND MATHEMATICAL MATTER}

These scientists support the idea of the universe being composed of information/mathematics:

a) In 1990, John Wheeler (1911-2008) suggested that information is fundamental to the physics of the universe. According to this "it from bit" doctrine, all things physical are information-theoretic in origin. (Wheeler, John A (1990) "Information, physics, quantum: The search for links". In Zurek, Wojciech Hubert. Complexity, Entropy, and the Physics of Information. Redwood City, California: Addison-Wesley)

b) Erik Verlinde says gravity is not a fundamental force of nature, but an emergent phenomenon. In the same way that temperature arises from the movement of microscopic particles, gravity emerges from the changes of fundamental bits of information, stored in the very structure of spacetime. (E P Verlinde (7 Nov 2016) "Emergent Gravity and the Dark Universe". arxiv.org/abs/1611.02269)

c) Cosmologist Max Tegmark hypothesizes that mathematical formulas create reality. (Max Tegmark (January 2014) "Our Mathematical Universe". Random House/Knopf)

d) "Pioneered (in the late 1980's) by Rafael Sorkin, a physicist at the Perimeter Institute in Waterloo, Canada, the theory (causal sets) postulates that the building blocks of space-time are simple mathematical points that are connected by links, with each link pointing from past to future." (Zeeya Merali (August 2013) "Theoretical physics: The origins of space and time". ("Nature" 500, 516-519-28))

If present-day physicists like Erik Verlinde and Max Tegmark are correct in thinking the universe has a mathematical foundation, that foundation could be the electronic BITS (BInary digiTS) of 1 and 0 , which comprise what is known as base- 2 mathematics. The 
exactness of binary calculation would then be the information which biochemist Christian Anfinsen determined was residing in the sequence of amino acids, and is necessary for protein folding. (Anfinsen, Christian B, Edgar Haber, Michael Sela, and F H White Jr. (15 September 1961) "The Kinetics of Formation of Native Ribonuclease During Oxidation of the Reduced Polypeptide Chain." Proceedings of the National Academy of Sciences of the United States of America 47, 9: 1309-1314) The exactness could also change present understanding of quantum mechanics by deleting probability in subatomic reactions - ensuring that if enzymes use quantum tunneling (where a particle passes through a barrier that it classically cannot penetrate) to accelerate biochemical reactions, (Jim Al-Khalili and Johnjoe McFadden (November 6th 2014) "Life on the Edge: The Coming of Age of Quantum Biology". First published by Bantam Press) those enzymes are guaranteed to function properly within the body and will not merely have a probability of being successful.

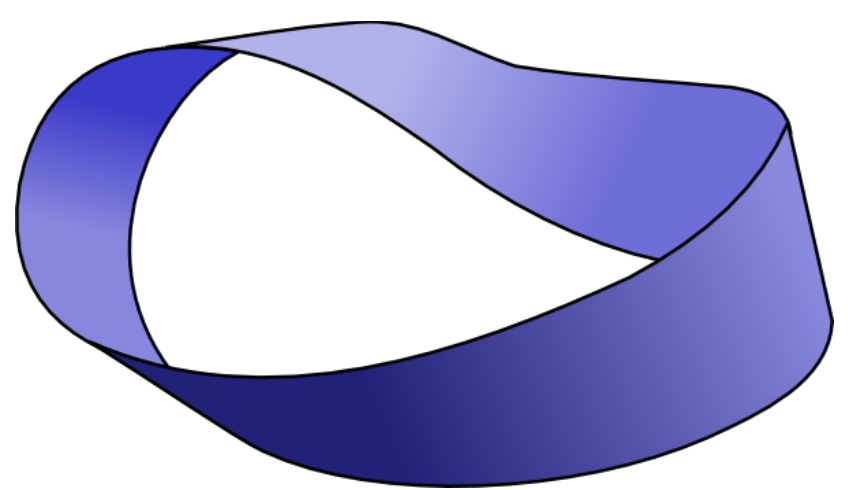

Figure 2 - the Mobius Strip, which is two-dimensional and only has one surface (source: http://www.clker.com/cliparts/3/7/ a/9/1220546534781713951lummie Mobius Strip.svg.hi.png)

How could this information in the form of binary digits produce specific shapes that fit together like a lock and key? First, the 1's and 0's form the shape of a Mobius strip, which is merely two-dimensional (2-D). To use words from a recent paper In a holographic universe, all of the information in the universe is contained in $2 \mathrm{D}$ packages trillions of times smaller than an atom. (Niayesh Afshordi, Claudio Corianò, Luigi Delle Rose, Elizabeth Gould, and Kostas Skenderis (27 January 2017) "From Planck Data to Planck Era: Observational Tests of Holographic Cosmology". Phys. Rev. Lett. 118, 041301. (https://journals.aps.org/prl/ abstract/10.1103/PhysRevLett.118.041301)) 
("Holographic" would have the accepted cosmological meaning of the entire universe being seen as two-dimensional information - from Mobius strips, according to this article - projected into the three dimensions we're familiar with.) Then two strips must be joined to make a Klein bottle (Polthier, Konrad (September 1, 2003) "Imaging maths Inside the Klein bottle". http://plus. maths.org/content/os/issue26/features/mathart/index) which has the 3 dimensions of length, width, depth - and the 4th dimension of movement in time. The type of Klein bottle formed would appear to be the figure-8 Klein. A diagram of many figure-8 Klein bottles would show that their positive curvature (on the spherical parts) fits together with their negative curvature (on saddle-shaped parts) to cancel and produce, on a cosmic scale, the flat curvature of space-time (WMAP science team (12/20/2012) "Wilkinson Microwave Anisotropy Probe".

https://map.gsfc.nasa.gov).

When you have trillions of Mobius and figure-8 Klein elements assembled, you can follow the theory of the mass-giving Higgs field being the result of various couplings. ( $M$. Tanabashi, M. Harada, and K. Yamawaki (2006) "The Origin of Mass and Strong Coupling Gauge Theories". "The Origin of Mass and Strong Coupling Gauge Theories". International Workshop on Strongly Coupled Gauge Theories.Nagoya. pp. 227-241) This theory has lost popularity since the Higgs boson was discovered. But an implication of a 1919 paper by Einstein called "Do gravitational fields play an essential role in the structure of elementary particles?" (Albert Einstein (1919) "Do gravitational fields play an essential role in the structure of elementary particles?" ("Spielen Gravitationsfelder im Aufbau der materiellen Elementarteilchen eine wesentliche Rolle?") Sitzungsberichte der Preussischen Akademie der Wissenschaften, [Math. Phys.], 349-356. Berlin) is that the coupling is between gravitons (the hypothetical quantum units of gravity) and photons (the quantum units of electromagnetism such as visible light, radio waves and $\mathrm{X}$-rays). That could mean coupling is also between the Mobius strips and figure-8 Klein bottles composing the particles. It only needs to be assumed that these topological shapes exist on a level between photons/gravitons and 1 's/0's, being built up into the particles and being composed of the binary digits). With trillions of Mobius and figure-8 Klein elements assembled, these (now respectively called photons and gravitons) must interact to give matter what we call the emergent property of mass - similar to hydrogen and oxygen combining to give water what we call wetness. This proposed link between the Mobius strip and the Mobius doublet (figure-8 Klein bottle) would also be a link between the photon and graviton - suggesting the unification of electromagnetism with gravitation, which Einstein sought in his Unified Field Theory (he died before completing the theory). It also confirms Erik Verlinde's idea that gravity is an emergent property (emerging from mathematics). 


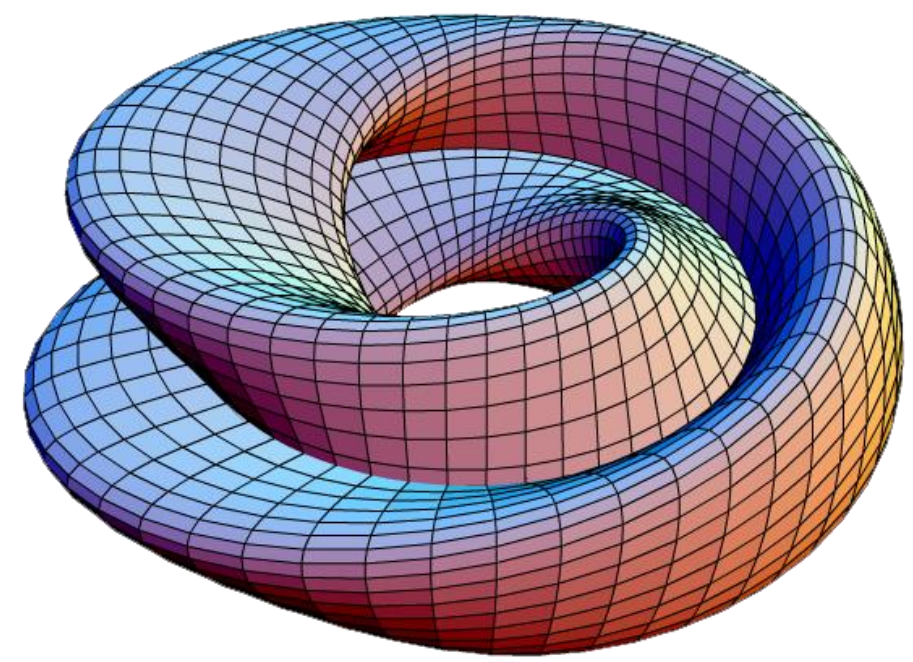

Figure 3 - MOBIUS DOUBLET (FIGURE-8 KLEIN BOTTLE) (source:

https://upload.wikimedia.org/wikipedia/commons/7/73/KleinBottleFigure8-01.png) Note that, when considering many bottles, the reddish positive curvature fits together with the bluish negative curvature to produce the flatness implying space-time's infinity and, since space and time are always unified, its eternity. (In flat space-time, light beams travel in straight lines and can go infinite distance without ever meeting.)

Following Albert Einstein's example of turning Max Planck's quanta (which, for years, Planck and all other scientists considered purely mathematical) into explanation of the physical photoelectric effect, the Wick rotation used to describe imaginary time may be transformed from mathematical "trickery" to physical meaning, and provide a modern way to unite space and time into one space-time. 


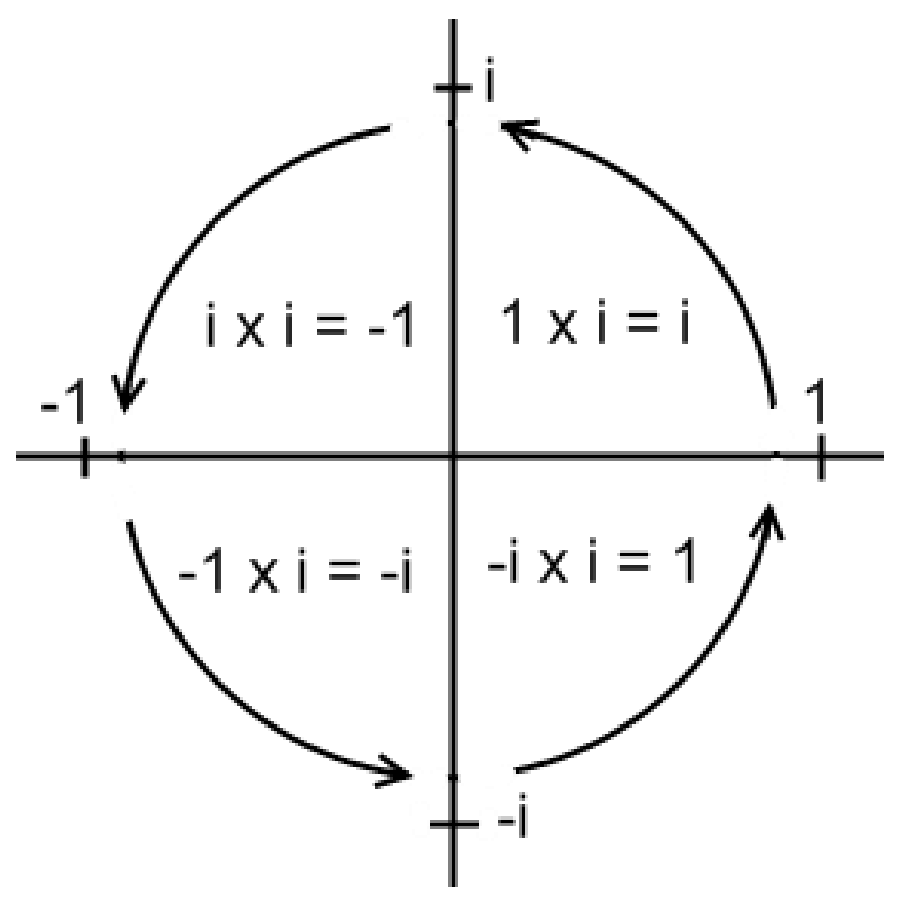

Figure 4 - WICK ROTATION: "The complex plane reveals i's special relationship with cycles via the circle of $i$, also known as Wick rotation. Whenever a point on the complex plane is multiplied by $\mathrm{i}$, it moves a quarter rotation around the origin or center of the plane." [Figure and quote from "The Meaning of Imaginary Time: Creativity's Dialog with Timelessness" - Posted on July 15, 2015 by Kerri Welch (public domain figure supplied by WordPress) https://textureoftime.wordpress.com/2015/07/15/the-meaning-ofimaginary-time/]

This submission claims Mobius strips compose energy particles as well as matter particles (see "Spin $1 / 2$ is like a Mobius strip" above, in VECTOR-TENSOR-SCALAR GEOMETRY AND THE NUCLEAR FORCES) and the Wick rotation inherent in the strips is adaptive. There are vastly increased numbers of gravitational energy's gravitons near black holes, and there is increased effect on electromagnetic energy's photons when speed-of-light travel includes vast numbers of photons in the traveller's sphere of influence. These enlarged quantities boost the probability of Wick rotations cancelling to produce quantum entanglement. "Advanced" waves travel back in time (to the left of Fig. 4's origin or centre). "Retarded" waves go forwards in time (to the right of Fig. 4's centre). Imagine the wave below as a retarded wave originating at the red dot on the far left. As it rotates around the origin in Fig. 4's anticlockwise direction to occupy coordinates proceeding to the left of the origin, its crests become troughs and troughs become crests (the other red dots mark the straight lines of the axes). In this way, the crests and troughs cancel each other and cancellation produces quantum mechanics' entanglement. In other words, a wave interferes with itself (and a particle - whether 
boson or fermion - formed from gravitational and electromagnetic waves interacts with itself). This interference of particles is demonstrated by the Mach-Zehnder interferometer since it splits light beams, causes travel in different directions, and recombines the photons. Interference of particles with themselves is also demonstrated by supersolids (solids that have superfluid - frictionless - properties and can flow through themselves). Their "superflow" (frictionless flow) may not be due to actual fluid moving along imperfections but to a special quantum state (where particles interfere with themselves) being formed at a low temperature very close to absolute zero.

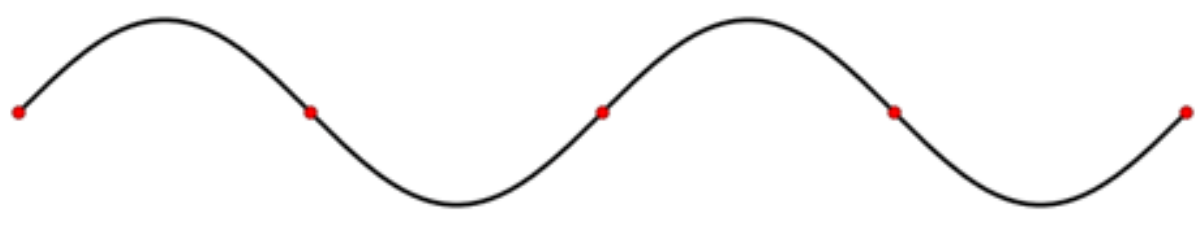

Figure 5 - Wave (Public domain image from https://en.wikipedia.org/wiki/Wave\#/media/File:Standing wave.gif)

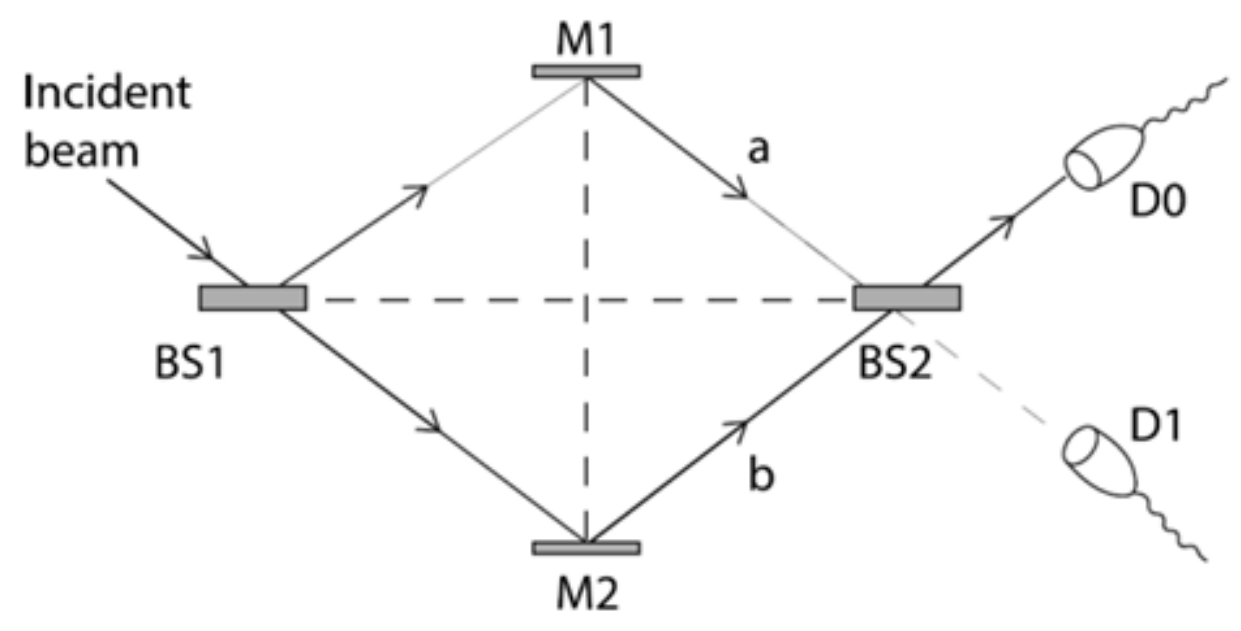

Figure 6 - Mach-Zehnder interferometer

We have two beam splitters (BS1 and BS2) and two perfect mirrors (M1 and M2). An incident beam coming from the left is split at BS1 and recombines at BS2, which sends two outgoing beams to the photon detectors D0 and D1. The interferometer can be set up to produce a precise interference effect which ensures all the light goes into D0, as 
shown above. Alternatively, the setup may be altered to ensure all the light goes into D1.

(Source of figure and figure's text - "Reading Feynman" by Jean Louis Van Belle: https://readingfeynman.org/tag/interference-of-a-photon-with-itself/. His reference for the illustration is MIT edX Course 8.04.1X (Quantum Physics), Lecture Notes, Chapter 1. Section 4 (Quantum Superpositions).

As well, an effect of gravitational and electromagnetic waves following Wick rotation is to cause more and more cancelling crests and troughs to interfere with themselves and produce Special Relativity's time dilation (slowing - with eventual stoppage at the speed of light) near the intense gravity of black holes, and near light speed. They also move the idea of waves travelling back in time into agreement with verified Special Relativity and quantum mechanics.

The Mobius Doublet (Figure-8 Klein Bottle) has space-time's four dimensions when adaptive Wick rotation is added to Mobius strips, manifesting as Special Relativity's non-absolute time. About the adaptive nature of Wick rotation: "Physicists now believe that entanglement between particles exists everywhere, all the time, and have recently found shocking evidence that it affects the wider, 'macroscopic' world that we inhabit" ("The Weirdest Link": New Scientist, vol. 181, issue 2440 - 27 March 2004, page 32 online at http://www.biophysica.com/QUANTUM.HTM). "Caslav Brukner, working with Vlatko Vedral and two other Imperial College researchers, has uncovered a radical twist. They have shown that moments of time can become entangled too" ("Quantum Entanglement in Time" by Caslav Brukner, Samuel Taylor, Sancho Cheung, Vlatko Vedral (Submitted on 18 Feb 2004) - http://www.arxiv.org/abs/quant-ph/0402127). When the figure-8 Klein bottles (which contain Wick rotation in their constituent Mobius strips) encounter other figure-8's, there's a probability that their rotations will cancel because of entanglement (one rotation may be clockwise with the other being anticlockwise), slowing and even stopping time. As the number of figure-8's accumulates, the chance of time's cessation increases. Think of a black hole as a focus for gravitational waves. The gravitons composing the waves are themselves composed of figure-8's. As these Klein bottles accumulate, time slows (the time dilation is lessened with bodies of reduced mass ie bodies such as stars and planets which are the focus of fewer, less powerful waves). In agreement with Relativity, intense gravity slows time. Speeds approaching $c$ (the velocity of light in a vacuum) do the same. Great speeds affect great numbers of Wick-rotation-containing Mobius strips and figure-8 Klein bottles (composers of, respectively, the universe's entanglement of photons and gravitons) resulting in increased chance of time slowing due to cancelling rotations. In conclusion; 
if gravitons and photons are entangled, so are the figure- 8 Klein bottles and Mobius strips and Wick rotations that compose the particles.

\section{ANALOGY OF QUANTUM SPIN AND MATRIX ARRAY, WITH ELECTROMAGNETIC AND GRAVITATIONAL WAVES PRODUCED FROM PURE MATHEMATICS}

This section was inspired by the astronomy/astrophysics course "Greatest Unsolved Mysteries of the Universe". It was prepared by ANU (the Australian National University), was put on the Internet by edX, and the two presenters in the course's videos are Dr. Paul Francis and Brian Schmidt (yes, the winner of the Nobel Prize for Physics himself).

In the video "V5.8: 21 centimetre radiation and the first stars", Brian said

"(To get emission from the hydrogen atom) we're going to have to resort to a little trick of quantum mechanics that is provided for the hydrogen atom, which is that the proton and electron within quantum mechanics have a spin. It's analogous to a top spinning. It turns out that the hydrogen atom has a different energy if those spins are in opposite directions. It turns out it actually has a lower energy and so you can have an energy transition when that flip occurs and a photon comes out."

Then Paul said,

"It's a bit like having two bar magnets. If you have them with north pole to north pole, or north pole to south pole, you'll feel a different amount of force between them; a different energy between these two things. When these things are spinning the same way or the opposite way there's a little tiny energy difference between them."

The following explains analogy of quantum spin to maths' matrix and how, using that analogy, the statements by Brian and Paul can be converted into a mathematical origin of electromagnetism and gravitation.

These five scientists support the idea of the universe being composed of information/mathematics: 
a) The digital physics pioneered by Professor Edward Fredkin believes that biology reduces to chemistry reduces to physics reduces to the computation of information. (Edward Fredkin, 'Digital Philosophy', http://www.digitalphilosophy.org/ (2013))

b) In 1990, John Wheeler (1911-2008) suggested that information is fundamental to the physics of the universe. According to this 'it from bit' doctrine, all things physical are information-theoretic in origin. (John A. Wheeler, 'Information, physics, quantum: The search for links', in Zurek, Wojciech Hubert, Complexity, Entropy, and the Physics of Information (Redwood City, California: Addison-Wesley 1990))

c) Erik Verlinde says gravity is not a fundamental force of nature, but an emergent phenomenon. In the same way that temperature arises from the movement of microscopic particles, gravity emerges from the changes of fundamental bits of information, stored in the very structure of spacetime. (E. P. Verlinde, 'Emergent Gravity and the Dark Universe', arxiv.org/abs/1611.02269 (2016))

d) Cosmologist Max Tegmark hypothesizes that mathematical formulas create reality. (Max Tegmark, Our Mathematical Universe (Random House/Knopf, 2014))

e) 'Pioneered (in the late 1980's) by Rafael Sorkin, a physicist at the Perimeter Institute in Waterloo, Canada, the theory (causal sets) postulates that the building blocks of space-time are simple mathematical points that are connected by links, with each link pointing from past to future.' (Zeeya Merali, 'Theoretical physics: The origins of space and time', Nature 500 (2013), 516-519)

The following explains how the statements by Brian and Paul can be converted into a mathematical origin of electromagnetism and gravitation.

First, let's look at the statement "... particles within quantum mechanics have a spin. It's analogous to a top spinning." The 2008 book "Quantum" by physicist and philosopher Manjit Kumar (Icon Books, pp. 382-383) says,

"Quantum spin is a fundamental property of particles with no direct counterpart in classical physics. Any picturesque comparison of a 'spinning' electron to a spinning top is merely a poor aid that fails to capture the essence of this quantum concept. The quantum spin of a particle cannot be explained in terms of classical rotation since it can only have certain values that are equal to a whole number or half a whole number multiplied by Planck's constant h divided by $2 \pi$ (a quantity called $\mathrm{h}$-bar). Quantum spin is said to be either up (clockwise) or down (anti-clockwise) with respect to the direction of measurement." 
I imagine the early part of this ANU course only needs a "picturesque comparison" nevertheless, that might be enough to demonstrate that we live in a universe composed of maths. My idea is that quantum spin may actually be analogous to the matrix, a rectangular array of numbers or symbols placed in rows and columns. Matrices have a long history possibly going back 3,000 years to their use in solving simultaneous equations in China. In the mid-nineteenth century, British mathematician Arthur Cayley discovered how to add, subtract, multiply and divide them. Matrix multiplication says $X$ multiplied by $\mathrm{Y}$ does not always equal $\mathrm{Y}$ times $\mathrm{X}$.

Suppose we think of the matrix array as composed of two bar magnets, with the bar magnets being composed of photons and gravitons instead of atoms (the energy and momentum of the photons and gravitons exert a pressure which we call mass when it's in particles or atoms). As Paul Camp, Ph.D. in theoretical physics, writes at https://www.quora.com/How-big-is-a-photon -

"A photon is a quantum of excitation of the electromagnetic field. That field fills all space and so do its quantum modes."

(Since matter exists in space, let's assume for the moment that the electromagnetic field and its photons also fill all matter - we can abandon this hypothesis later if it fails). Why photons AND gravitons? Mathematician and physicist Ron Kurtus writes at http://www.schoolforchampions.com/science/gravitation electrostatic.htm\#.Wkw9dcs 5Ah

(5 December 2010) - "An analogy of gravitational and electromagnetic fields is seen by comparing the Einstein Field Equations from the General Theory of Relativity with Maxwell's Field Equations for electrical and magnetic fields."

Now let's return to "Matrix multiplication says $X$ multiplied by $Y$ does not always equal $Y$ times $X "$ and "the matrix array (being) composed of two bar magnets". $X Y$ can represent magnetic North on one bar attracting magnetic South on the other. YX can represent South repelling South. When different magnetic poles (or different combinations of the two letters) are close together, you'll feel a different force between them - there's a little tiny energy difference between them. Picture the bar magnets as coming together at an angle - not in a straight line. When quantum spin changes from XY's attraction to YX's repulsion, nano-nano-nano sized bits of the magnets (the photons and gravitons composing them) are repelled or emitted, accounting for electromagnetic and gravitational waves from the magnets. In reality, the magnets are merely a substitute for the matrix array which appears to truly be analogous to quantum spin and also appears to provide a purely mathematical origin for electromagnetic and gravitational waves. 
DARK MATTER, DARK ENERGY AND A HIGHER DIMENSION OF SPACE-TIME

This part deals with mathematics similar to the matrix, a rectangular array of numbers or symbols placed in rows and columns. For example, the underlined entry 2340 in the product is calculated as $(2 \times 1000)+(3 \times 100)+(4 \times 10)=2340$ :

$$
\left[\begin{array}{lll}
\underline{2} & \underline{3} & \underline{4} \\
1 & 0 & 0
\end{array}\right]\left[\begin{array}{ll}
0 & \underline{1000} \\
1 & \underline{100} \\
0 & \underline{10}
\end{array}\right]=\left[\begin{array}{ll}
3 & \underline{2340} \\
0 & 1000
\end{array}\right] .
$$

Figure 7 - Matrix multiplication

From https://en.wikipedia.org/wiki/Matrix (mathematics) This Wikipedia reference is not used to support a scientific claim, but merely as an example of what basic matrix multiplication looks like.

Matrix mechanics is a version of quantum mechanics discovered by Werner Heisenberg in 1925, and matrix multiplication says $X$ multiplied by $Y$ does not always equal $Y$ times $X$. The book "Quantum" states, Max Born wrote to Albert Einstein that "Heisenberg's latest paper, soon to be published, appears rather mystifying, but is certainly true and profound." He was referring to "the strange multiplication rule" Heisenberg used in developing matrix mechanics. Born eventually realised that Heisenberg had stumbled on matrix multiplication - to which the originator of matrix mechanics replied, "I do not even know what a matrix is." (Cropper, William H. [2001] "Great Physicists: The Life and Times of Leading Physicists from Galileo to Hawking" - Oxford: Oxford University Press, p. 269) 


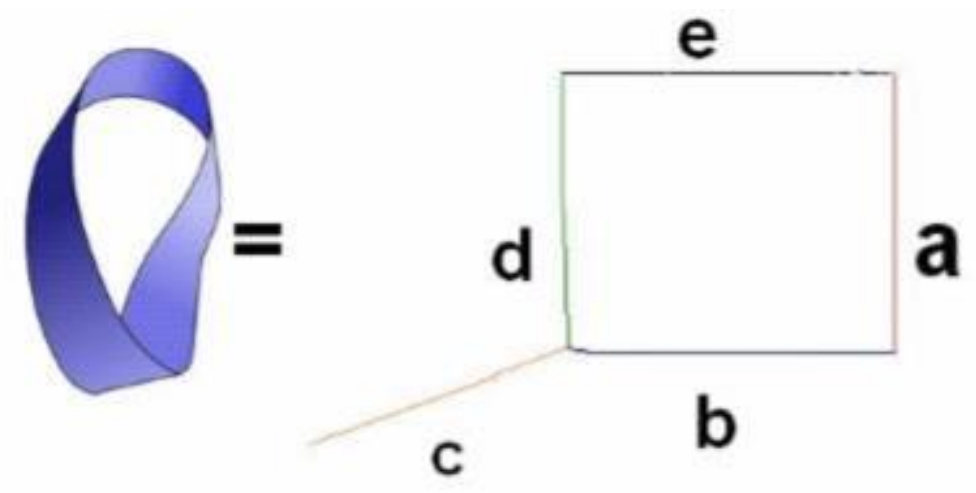

Figure 8 - MOBIUS MATRIX (Mobius equals a,b,c,d,e array)

Width $\mathrm{a}$ is perpendicular to the length ( $\mathrm{b}$ or $\mathrm{e}$ ) which is perpendicular to height $\mathrm{c}$. How can a line be drawn perpendicular to $c$ without retracing b's path? By positioning it at $d$, which is then parallel to (or, it could be said, at 180 degrees to) a. $d$ is already at 90 degrees to length $b$ and height $c$. $d$ has to be at right angles to length, width and height simultaneously if it's going to include the Complex Plane's vertical "imaginary" axis in space-time (the "imaginary" realm is at a right angle to the 4 known dimensions of space-time, which all reside on the horizontal real plane). In other words, $d$ has to also be perpendicular to (not parallel to) a. This is accomplished by a twist, like on the right side of the Mobius strip, existing in the particles of matter composing side a. In other words, matter's most fundamental composition is mathematics' topological Mobius, which can be depicted in 3-dimensional space by binary digits creating a computer image). The twist needs to be exaggerated, with the upper right of the Mobius descending parallel to side "a" then turning perpendicular to it at approximately the level of the $=$ sign, then resuming being parallel. Thus, $90+90$ (the degrees between b \& c added to the degrees between $c \& d$ ) can equal 180, making a \& d parallel. But $90+90$ can also equal 90 , making a \& d perpendicular. (Saying $90+90=90$ sounds ridiculous but it has similarities to the Matrix [of mathematics, not the action-science fiction movie] in which $X$ multiplied by $Y$ does not always equal $Y$ times $X$. The first 90 plus the second 90 does not always equal the second 90 plus the first 90 because $90+90$ can equal either 180 or 90 .

(1) The matrix mathematics/matrix mechanics of $90+90$ equalling either 180 or 90 can also be related to the fact that a neutron bound in an atom's nucleus can be stable but a free neutron is unstable - decaying to a proton, electron and antineutrino with an average lifetime of just under 15 minutes. $90+90=180$ possesses a change to the numerals and is likened to instability while $90+90=90$ has no change and has analogy to 
stability. Why aren't protons or electrons (for example) affected? Since protons bound in a nucleus can be stable or unstable (protons can transform into neutrons which can change into protons since neutrons can be unstable), the matrix maths/mechanics must be affecting them - the influence on neutrons may be more apparent since these particles decay into protons and electrons. Neutrons bind with protons and one another in the nucleus via what is called the strong nuclear force, effectively moderating the repulsive forces between the protons and stabilizing the nucleus (radioactive decay proceeds via what's called the weak nuclear force so as to change the neutron/proton ratio to increase stability). This binding via the nuclear force can be regarded as an attractive force coming from the neutrons. Neutron formation, like formation of every particle, depends on gravitation interacting with electromagnetism (see Footnote 2). Therefore, it'd be correct to view the nuclear forces as non-fundamental and a product of gravitational force, just as the tides in Earth's oceans may be viewed as increased or reduced suppression of water waves by gravitational waves (Footnote 3 ).

(2) The matrix mathematics/matrix mechanics of $90+90$ equalling either 180 or 90 can also be related to the fact that the most abundant form of iron (atomic mass unit $=56$, comprises $92 \%$ of natural iron) is the most stable element, while there are unstable and radioactive isotopes with half-lives varying from nanoseconds to 2.6 million years. This might be able to be applied to the question's fusion of hydrogen into uranium. When fusion reaches the most stable element, it might be made to bypass that stability and continue all the way to uranium. How? By using that higher dimension - which might possibly be the origin of dark matter and dark energy ${ }^{\wedge}$ - and manipulating the Mobius strip (space-time's topology). The manipulation might change the stability of iron's $90+90=90$ into the instability of $90+90=180$, and that instability should allow fusion to continue far beyond the cobalt and nickel that decay to iron.

(3) According to the stress tensor of vector-tensor-scalar geometry, both matter and dark matter can be formed by the interaction of gravitation and electromagnetism. The only difference between them would be that dark matter is, to use Wick rotation, rotated 90 degrees from ordinary matter's horizontal $x$-axis to the vertical, "imaginary" $y$-axis. It's rotated into another dimension. Since this rotation twists the electromagnetic spectrum perpendicular to our perceptions and instruments, the dark matter is only detectable gravitationally (because it still resides in space-time and General Relativity says gravity is the curvature of space-time; therefore, gravity is space-time). Though united with this dimension, it may be visualized as existing "above" and "below" ordinary space-time: in "hyperspace" and "subspace". Following Einstein's $E=m c^{2}$, the relation between Dark Matter (DM) and the Dark Energy it possesses (DE) would be DE=DMc ${ }^{2}$. In 
conclusion; if the gravitons and photons are entangled, so are the figure-8 Klein bottles and Mobius strips and Wick rotations that compose the particles.

Mathematics has three types of numbers - real, imaginary and complex. Real numbers are exemplified by 0 , the positive numbers used in counting and negative numbers. On a two dimensional "Complex Plane", 'Real Numbers' are on the horizontal plane and 'Imaginary Numbers such as $i=\sqrt{ }(-1)$ are on the vertical plane. 'Complex Numbers' can be easily identified as a combination of 'Real Numbers' and 'Imaginary Numbers'. ("Difference Between Complex Numbers and Real Numbers" - June 18, 2011 - Posted by Olivia https://www.differencebetween.com/difference-between-complexnumbersand-vs-real-numbers/) Retarded gravitational and electromagnetic waves that go forwards in the horizontal plane of space-time can be termed real. Advanced waves that go backwards in space-time may be considered complex. The imaginary numbers of the vertical direction could describe waves in an "imaginary" space-time.

On the subject of dimensions of space-time: Professor Itzhak Bars of the University of Southern California in Los Angeles says,

'one whole dimension of time and another of space have until now gone entirely unnoticed by us'. (Tom Siegfried, 'A Two-Time Universe? Physicist Explores How Second Dimension of Time Could Unify Physics Laws', May 152007 https://m.phys.org/news/2007-05-twotime-universe-physicist-exploresdimension.html)

Could Prof. Bars' second dimension of space be imaginary (in the sense of $i=\sqrt{ }-1$ ) space which is united with imaginary time the same way ordinary space and time are joined? And in the unification of a quantum gravity universe, the real and imaginary would be connected (quantum gravity is the anticipated unification of quantum mechanics with Einstein's theory of gravity - General Relativity).

We see that the Mobius is necessary to the immaterial portions of the world - not only undetected large-scale dimensions but also particles of energy (the photon of electromagnetism would be an assembly of trillions of strips). The subject of quantum spin and Wolfgang Pauli will be mentioned now. In relation to Quantum Spin, Wolfgang Pauli in 1924 was the first to propose a doubling of electron states due to a two-valued non-classical "hidden rotation". Extending the ideas of "doubling", "two- 
valued" and "hidden rotation" to the Mobius strip being a basic, fundamental unit of reality; it can be seen that Pauli's proposal has an analogy to this article. The doubled Mobius strips produced by the two-valued binary-digit system create the figure-8 Klein bottle (plus its hidden, now identified as Wick, rotation). This not only unites the Mobius strip with the figure-8 Klein bottle but also the photon with the graviton ie electromagnetism with gravitation. It also confirms Erik Verlinde's idea that gravity is an emergent property (emerging from mathematics).

\section{EXPLAINING OCEAN TIDES WHEN GENERAL RELATIVITY SAYS GRAVITY IS A PUSH CAUSED BY THE CURVATURE OF SPACE-TIME}

How, then, can repelling or pushing gravity account for the apparent attraction of ocean tides towards the Moon? I believe such an idea of gravity requires the idea of $17^{\text {th }}$-century scientists Isaac Newton and Johannes Kepler that the moon causes the tides, to be joined with Galileo's idea that the Earth's movements slosh its water.

"If a barge (carrying a cargo of freshwater) suddenly ground to a halt on a sandbar, for instance, the water pushed up towards the bow then bounced back toward the stern, doing this several times with ever decreasing agitation until it returned to a level state. Galileo realized that the Earth's dual motion -its daily one around its axis and its annual one around the sun-might have the same effect on oceans and other great bodies of water as the barge had on its freshwater cargo." (Tyson Peter, "Galileo's Big Mistake" Posted 10.29.02 (http://www.pbs.org/wgbh/nova/earth/galileo-big-mistake.html))

Gravity's apparent attraction can be summarized by the following - gravitation is absorbed into wave packets and the inertia of the gravitons (united with far more energetic photons) carries objects towards Earth's centre at $9.8 \mathrm{~m} / \mathrm{s}$ or $32 \mathrm{ft} / \mathrm{s}$. The volume of the oceans on Earth is estimated at nearly 1.5 billion cubic kilometres. (Van Nostrand's Scientific Encyclopedia, 10th edition, "Ocean Volume and Depth" 2008) All this water is being pushed towards Earth's centre at 32 feet per second every second. But the seafloor prevents its descent. So there is a recoil, noticeable offshore (it is only where oceans and continents meet that tides are great enough to be noticed). This recoil is larger during the spring tides seen at full and new moon because sun, Earth and moon are aligned at these times. 
The previous paragraph's alignment of Sun, Earth and moon refers to their being lined up where the gravitational current is greatest (in the plane where planets and moons are created) - and to more of the gravitational waves travelling from the outer solar system being captured by solar and lunar wave packets, and less of them being available on Earth to suppress oceanic recoil (there are still enough to maintain the falling-bodies rate of 32 feet per second per second). At the neap tides of $1 \mathrm{st}$ and $3 \mathrm{rd}$ quarter; the sun, earth and moon aren't lined up but form a right angle and our planet has access to more gravity waves, which suppress oceanic recoil to a greater degree. We can imagine the sun and moon pulling earth's water in different directions at neap tide but suppression is a more accurate description. If variables like wind/atmospheric pressure/storms are deleted, this greater suppression causes neap tides which are much lower than spring tides.
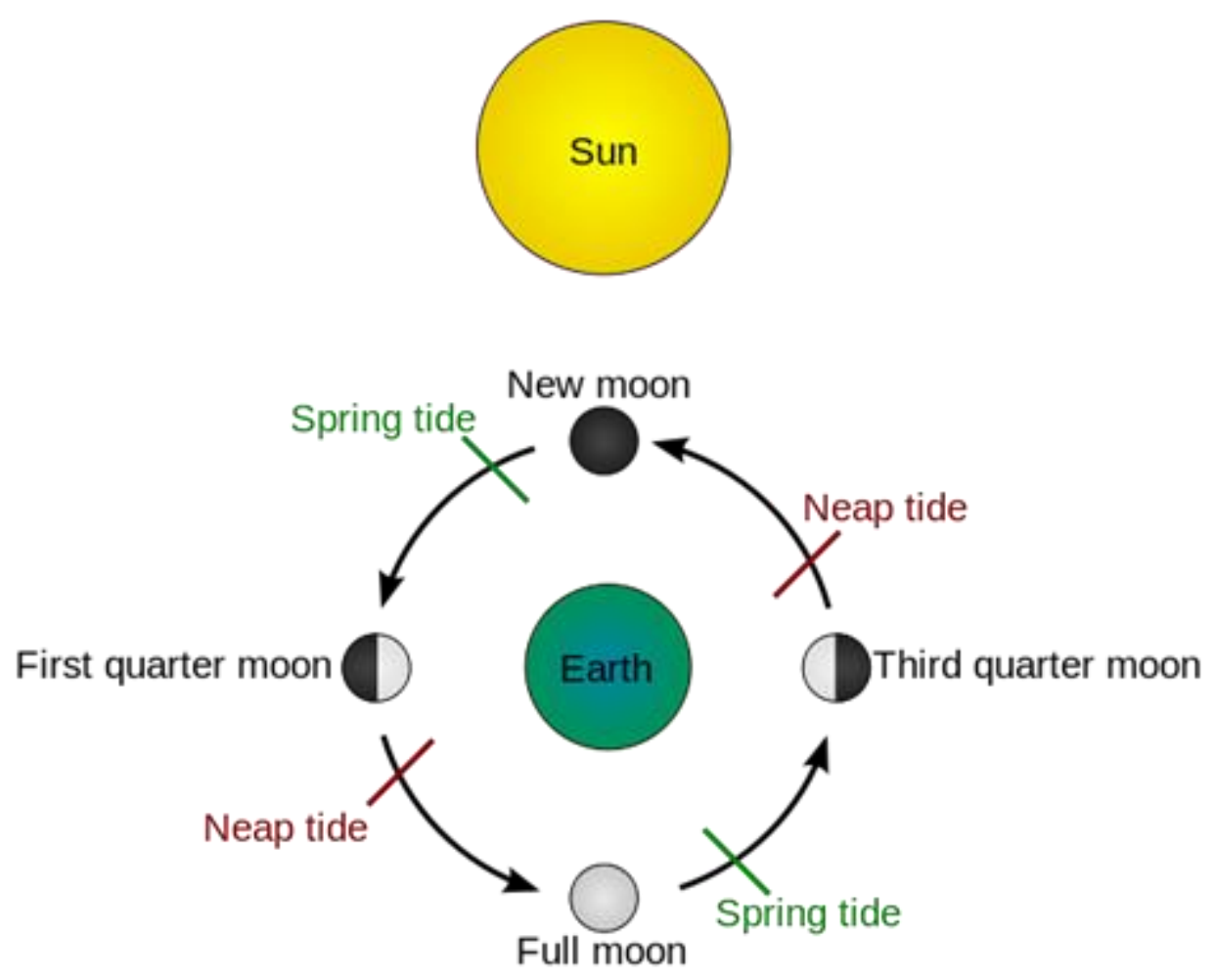

Figure 9 - TIDE SCHEMATIC

public domain image from https://en.wikipedia.org/wiki/File:Tide schematic.svg

Let's apply this aspect of gravity to a few other instances - 


\section{M-SIGMA}

The M-sigma relationship was only discovered in 2000 and is observational, meaning scientists noticed it first and are now trying to understand the cause. $M$ refers to the mass of a galaxy's central black hole, and sigma stands for the speed at which stars fly about in the galaxy's bulge. The bigger the black hole, the faster the stars move - the greater is their velocity dispersion. ("The M-sigma relationship": Astronomy, October 2016)

Gravitational waves would explain the cause. Some of the ocean waves passing an island are refracted - when they enter shallow water, they're refracted by friction with the mass of the seabed. They change direction and head towards the island, breaking onto its beaches. Similarly, gravitational waves are refracted and focus on the centre of a mass. In this case, the mass the waves are headed toward is the black hole, where they help form its composition.

General Relativity proposes that the space-time composing the cosmos IS gravitation. Gravitational waves not only compose space-time but also so-called "imaginary" spacetime (which exists on the Complex Number Plane's y-axis, can interact with our dimension on the $x$-axis, and is the possible domain of what are called dark matter and dark energy). The linear motion of waves headed towards the central black hole and striking stars' sides during the journey is converted into increased (and perpendicular) orbital speed of the stars since the gravitational waves of imaginary time are at 90 degrees to the gravitational waves of space-time (recall how we can picture imaginary time as another kind of time in the vertical direction when familiar time is a horizontal line).

\section{GEYSERS ON SATURN'S MOON ENCELADUS}

"A small water jet on Enceladus, an icy moon of Saturn, spews its fiercest eruptions when the moon is farthest from the planet, a new study suggests, but the overall gas output doesn't increase much during that time. The study points to a mystery in Enceladus' 
plumbing." ("Surprising geysers on Saturn moon Enceladus hint at plumbing mystery" by Elizabeth Howell, Space.com Contributor . Published May 12, 2016 (http://www.foxnews.com/science/2016/05/12/surprising-geysers-on-saturn-moonenceladus-hint-at-plumbing-mystery.html))

Basically, the problem seems to be that humans haven't caught up with Einstein's ideas about gravity yet. In 1919, he submitted a paper to the Prussian Academy of Sciences asking "Do gravitational fields play an essential role in the structure of elementary particles?" (Albert Einstein's "Spielen Gravitationfelder in Aufbau der Elementarteilchen eine Wesentliche Rolle?" (Do gravitational fields play an essential role in the structure of elementary particles?), Sitzungsberichte der Preussischen Akademie der Wissenschaften, (Math. Phys.), 349-356 (1919) Berlin]) If so, gravitational waves from deep space would focus on the centre of a planet's mass. When Enceladus is near Saturn, it would also be close to increased activity of the waves. The increased push from them would suppress emission of dust-sized water-ice grains, which is 3 times greater at the moon's farthest point because suppression is reduced there. Gas emission is also increased. Since this is not 3 times more, but only $20 \%$ more, a plumbing problem would be causing the discrepancy.

\section{A BRIEF HISTORY OF GRAVITY}

In three dimensions, the gravitational force drops to $1 / 4$ if one doubles the distance. In four dimensions (4th-dimensional hyperspace), it would drop to $1 / 8$ and in five dimensions (5th-dimensional hyperspace) to 1/16: "A Brief History of Time" by Stephen Hawking Bantam Press 1988, pp. 164-165. The positive direction on the x-axis (representing the 3 space dimensions of "real" space-time) is in continuous contact with the negative direction on $x$ (this may be called the 5th space dimension, complex space-time, providing access to the past). Therefore, real gravity is perpetually amplified by complex gravity. Using Professor Hawking's figures, the amplification equals $1 / 4 \times 1 / 4$ ie doubling the distance in 5 space dimensions causes gravity to become $1 / 16$ as powerful. It is not $1 / 4 \mathrm{X}$ $-1 / 4$ since numbers have the same property regardless of direction on the Complex Number Plane (they increase in value). To conserve this sameness, the second one must be $+1 / 4$ if the first one is $+1 / 4$. Alternatively, the gravity's strength is reduced 4 times and this number is multiplied by another 4 to reduce it 16 times overall. In the 4th space dimension/2nd time dimension represented by the imaginary axis, this y-axis is half the 
distance (90 degrees) from the real $x$-axis that the complex x-axis is (it's removed 180 degrees). So gravitational weakening from doubling distance in 4 space dimensions = (reduction of 4 times multiplied by another reduction of 4 times) / 2 , for an overall reduction of 8 times to a strength of $1 / 8$. 